\title{
Design of an Appliance Level Eco-Feedback Display for Domestic Electricity Consumption
}

\author{
Jeni Paay, Jesper Kjeldskov, Mikael B. Skov, Dennis Lund, Tue Madsen, Michael Nielsen \\ Research Centre for Socio+Interactive Design \\ Department of Computer Science, Aalborg University, Denmark \\ $\{$ jeni, jesper,dubois\}@cs.aau.dk
}

\begin{abstract}
Over the past decade there has been an increased focus on eco-feedback systems for electricity consumption due to emerging technologies that allow detailed and real-time usage data to be collected and presented to users. In this paper, we present the design of an always-on ecofeedback display, PowerViz, that provides information about people's power usage in their homes at an appliance level. In our study, we found that PowerViz increased awareness towards energy consumption, gave householders a better understanding of high consumption devices and made it easy for them to isolate and respond to unnecessary or "greedy" appliances by turning them off or changing their use patterns. We also found that an ambient display of the household's total current electricity use both informed and attracted people to use the system when power usage became unusually high.
\end{abstract}

\section{Author Keywords}

Eco-feedback; Electricity; Sustainability; Design; Technology probe; Always-on display; Situated display;

\section{ACM Classification Keywords}

H5.m. Information interfaces and presentation (e.g., HCI): Miscellaneous.

\section{INTRODUCTION}

In recent years there has been an increased public concern for the environment (Froehlich, 2009). Heavy use of the world's non-renewable resources combined with an increase in pollution has prompted an increased interest in sustainability research. As the demand for more and more electronic devices increases worldwide, there is an increasing need for research into supporting sustainable electricity consumption practices. The design and use of interactive technology that supports increased awareness of power use in the home is a current area of interest in HCI research (Schwartz et al., 2013). As one of the highest per capita consumers of energy, around $40 \%$ of all energy consumed in the US occurs in the domestic sector (EIA, 2013). This equals an average electricity consumption per household of around $11.8 \mathrm{MWh} /$ year. By comparison, the average household electricity consumption for Australian households is around 7

Permission to make digital or hard copies of all or part of this work for personal or classroom use is granted without fee provided that copies are not made or distributed for profit or commercial advantage and that copies bear this notice and the full citation on the first page. Copyrights for components of this work owned by others than ACM must be honored. Abstracting with credit is permitted. To copy otherwise, or republish, to post on servers or to redistribute to lists, requires prior specific permission and/or a fee. Request permissions from Permissions@acm.org.

OzCHI '14, December 03 - 05 2014, Sydney, NSW, Australia Copyright 2014 ACM 978-1-4503-0653-9/14/12 ..\$15.00 http://dx.doi.org/10.1145/2686612.2686663
$\mathrm{MWh} /$ year, and in Denmark $4 \mathrm{MWh} /$ year (World Energy Council, 2014). This shows that a significant contribution to reducing worldwide energy consumption can be made in the domestic sector in these countries, if people are both committed to sustainability and understand how they can contribute to savings. This makes research into HCI and sustainable technologies highly relevant for domestic electricity consumption.

One important way of achieving sustainable behaviour change is to provide better feedback on energy consumption (eco-feedback) to householders (Froehlich et al., 2010). A big part of the problem with reducing electricity consumption in our daily lives is that we are often unaware of the extent of our immediate consumption of energy and are only reminded when the quarterly bill comes in. By then it is difficult to understand how to change our behaviour for future sustainability if we cannot actually remember exactly what we did to achieve this total power use. Additional to this problem, for most people, information about current use of electricity in their home is hidden in a cupboard or in power box outside, and is presented as a myriad of undecipherable dials (Darby, 2005).

It is obviously a challenge to decrease domestic electricity use if people do not have knowledge about the effect of their behaviour and habits. Also, we cannot expect to support reduced electricity consumption without knowing more about the effects of systems designed to show power consumption patterns. Advances in technology make it possible to gather consumption data and present it to the members of a household in various ways other than on a meter in the cupboard. This makes it possible to give households the information needed to increase their awareness about their consumption, and potentially work toward changing their consumption behaviours. Through saving electricity they can show their commitment to the environment or simply save money. However, the interaction design of these systems to help users understand their use of electricity and enable them to act differently is challenging. How these systems should actually present this information to users and how people should interact with them to best effect is not yet well understood, especially the long-term effects of such systems (e.g., Cowan et al., 2013; Foster et al., 2011; Kjeldskov et al., 2012; Petkov et al., 2011; Schwartz et al., 2013). Research has shown that it is difficult to provide lasting engagement with these systems, and that to have the most impact on the householder and their practices over time, it is the usability of the device and its ability to provide meaningful information that are the most important (Snow et al., 2013). 
This paper presents the design, implementation, and field study of PowerViz, an always-on eco-feedback display, intended to increase consumers' awareness about electricity usage at an appliance level consumption. The purpose of the PowerViz system is to generate awareness of power consumption. This is done through several dimensions including making information about power use easily accessible to members of the household and that providing a variety of different visualizations.

PowerViz was deployed in three Danish households for seven weeks to explore people's reactions to, reflections on, and use of it. From this field study, we found that breaking consumption information down to appliance level, rather than the overall electricity consumption of the household, helped people to better understand the large amount of information available. The visualizations, providing information about current and recent consumption of the household at appliance level, made it easy for householders to react to this information and change electricity consumption behaviour in response to their everyday energy use. The always-on ambient display design of the screen saver, showing overall consumption, helped maintain an awareness of, and interest in the system.

\section{RELATED WORK}

Within the broader topic of environmental sustainability in HCI, two approaches to design have been identified: sustainability in design and sustainability through design (Mankoff et al., 2007). Sustainability in design describes approaches where the aim is to build technologies that are themselves more sustainable, for example, energy efficient devices. Sustainability through design describes the design of information technologies that support more sustainable human behaviour, for example, systems that show information about resource consumption to the consumer. Sustainability research in HCI falls primarily with in the latter of these two categories. Much of the research focuses on the design of information technologies, "eco-feedback" technologies or systems that can help people reduce energy consumption and promote more sustainable behaviour. Froehlich et al. (2010) define eco-feedback technologies as "technology that provides feedback on individual or group behaviours with a goal of reducing environmental impact". To achieve this goal, information should be presented by the system to users in an understandable and actionable way.

A variety of technologies have been utilized to explore areas such as electricity and water consumption. DiSalvo et al. (2010) map the landscape of sustainable HCI, giving an overview and critique of the various techniques used. They divide previous research into genres and show that $45 \%$ of published work uses pervasive technologies, and about $25 \%$ use ambient awareness. They also show that the majority of systems are aimed at individual consumers, rather than entire households or communities.

Fitzpatrick and Smith (2009) define a set of design concerns to frame and focus ongoing research on the design of the user experience and effectiveness of feedback displays. They review real-time, domestic energy displays to generate their concerns and show that multiple factors such as metrics, frequency and granularity play a role in the design of data feedback. They found that $49 \%$ of participants want real-time updates, and $28 \%$ of participants want to know when something has changed. They conclude that real-time feedback helps raise consumer awareness. They say that the feedback device, both in terms of form and location, should be placed in the home in a situation where people are able to glance at it when needed, or even when just passing by. They also say that considerations about how the device aesthetically blends in with the design of the home are equally important. Fitzpatrick and Smith conclude that technology-enabled feedback is well accepted as an information source to promote sustainable behaviour.

Froehlich et al. (2010) look at the design of eco-feedback technology combining both HCI and environmental psychology perspectives, specifically the role that HCI can play in the design of eco-feedback technologies. They suggest that HCI researchers should draw on the 40 years of work done by environmental psychologists in this area to avoid redundant efforts. They point out that with the advent of "smart meters", providing real-time or near real-time data, systems are more able to easily make consumers aware of their current consumption. Froehlich et al. claim that data granularity, time granularity and units of measurement are important when designing ecofeedback displays. Like Fitzpatrick and Smith (2009) they also found that the placement of the display within the home is important since information must be accessible to the household where and when needed it is needed.

From our review of current eco-feedback systems we found that technologies could be divided into two categories: 1) individual commitment, and 2) social commitment. Individual commitment is about making individuals feel responsible for their own actions towards the environment and becoming sustainable. Social commitment involves making groups of people feel responsible for their actions towards the environment.

We found that systems supporting individual commitment tended to use the following technologies: mobile applications, general applications, web portals, and hardware devices. The following are examples that illustrate each technology type we identified. Power Advisor (Kjeldskov et al., 2012) is a mobile application. It displays the electricity consumption of a single household on a mobile phone and gives feedback on, and recommendations about their consumption. Electricity consumption is shown as a graph of kilowatt-hours used over time. In addition to this, the same information is displayed in several different, and sometimes playful pictorial forms. For example, smiley faces that get grumpy the more power used, power gauges that move from green zones to red zones of the dial if the power use goes above the average, and graphical comparisons of current use against local use or personal pre-set goals. POEM (Personal Office Energy Monitor) (Milenkovic et al., 2013) is a general application that runs on desktop computers. It provides personalized data on electricity usage for a workplace. It also reports aggregates for 
building level management. Power Ballads (Foster et al., 2011) is a web portal. It makes use of social media, using it to provide negative feedback on non-desirable behaviour, which reportedly increases user engagement with the system. The Stroppy Kettle (Cowan et al., 2013) is a hardware device. As the name indicates, it is an ordinary looking kettle that can be used to boil water. It targets users' habitual kettle overfill behaviours and tries to affect behaviour change, by punishing them with a boring and time consuming task if they overfill the kettle.

For social commitment systems we found systems using situated displays, general applications, web portals, and hardware devices. The following examples illustrate each identified technology type. Always-on situated displays were the most prevalent in this category. This is where individual households have access to their domestic consumption data on a display placed in their home. There was no general form for these displays, but they were all running some sort of visualization software on a display. Some were interactive and others were purely informative. For example, the prototype fixture-level water usage data screens by Froehlich et al. (2012) provided a collection of creative visualizations and locations within the home to study their impact on family life. General applications, such as those being tested in living labs by Schwarz et al. (2013), present real-time data to households and study their increase in "energy literacy" over time. These are often classified as home energy management systems. The Dubuque Electricity Portal (Erikson et al., 2013) is a web portal. It provides fine-grained feedback on total electricity usage of a single household in respect to other households in that city. In this situation, a number of households have access to data about each other. The system uses incentives, social comparisons, and goal-setting to support behaviour change. The PowerSocket (Heller and Borchers, 2011) is a wall mounted power socket that visualizes the power consumption directly on the outlet, for all family members to see. Although this system can give appliance level feedback, it is only displayed locally on that power socket, so it is impossible to get an overview of the contribution to the overall consumption level, or any data of consumption over time with this system.

In our review, we distinguish between two types of data visualization: pragmatic and artistic (Pierce et al., 2008). Pragmatic visualizations are designed to remove any subliminal meanings and foster immediate understanding. Artistic visualizations keep the audience interested by showing data in an abstract way. For example, a pragmatic visualization of electricity consumption could be a graph that displays kilowatt-hours used over time (e.g. Froehlich et al., 2012; Kjeldskov et al., 2012; Milenkovic et al., 2013; Schwarz et al., 2013). Alternatively, Kim et al. (2009) use an artistic representation of power consumption in their system, Coralog, which displays a coral reef that changes colour and condition according to electricity usage.

A general tendency that we noticed in the literature was that studies of eco-feedback technologies have short evaluation periods. Most of the research systems surveyed had been deployed for less than three months. Riche et al. (2010) argue that new behaviours adopted during a research study have a tendency to relapse shortly after the study finishes, unless those changes have been adopted for at least three months. To counteract this, Riche et al. propose a three-staged approach to support user behaviour change: raise awareness, inform complex changes, and maintain sustainable routines. Our current study tackles the first stage of this approach, to raise awareness. Riche et al. advise that to achieve raised awareness in users, they need to obtain knowledge of their own electricity consumption patterns. To facilitate this, our system PowerViz provides householders with current and recent consumption patterns, visualised at appliance level, using real-time information.

\section{POWERVIZ}

The design of PowerViz was an iterative process, inspired by the design process presented in Froehlich et al. (2012). Froehlich et al. establish that iterative design is well suited to creating eco-feedback displays. Through a process of sketching, team design reviews, and redesigns, we created a final design.

In designing our information representations, we were guided by Froehlich at al. (2012) and Riche et al. (2010) on how to represent comparisons of consumption. Both agree that there are multiple ways to compare. Basically, comparisons can be made on an individual level, using historical data or pre-set goals, or at a social level, by making relative comparisons to energy consumption in similar households or community averages.

It is important that information presented to users is easy to understand (Richie et al., 2010). This is why we use pragmatic representations for ease of legibility of detailed appliance level information. At the same time, artistic visualizations help keep users interested in using a system (Pierce et al., 2008). Therefore, we use an artistic representation of overall consumption in the form of hanging light globes on the display's screen saver to maintain interest with the system. Changes in overall consumption of the household are continually updated using real-time data, and represented on the always-on screen saver as in increasing or decreasing number of light globes. This information is displayed in an ambient manner, allowing users to glance at the image to get an impression of energy use while engaged in other activities, thus blending with everyday activities of the household.

Information about energy consumption exists at different levels of granularity (Erickson et al., 2013; Froehlich et al., 2012; Riche et al., 2010). Data granularity is related to how data is sub-dived or grouped. Time granularity relates to the time period for which information is represented. Design decisions need to be made about which representations are appropriate in which situations. In terms of user engagement, there should be some elements in the application, which can draw the user's attention and keep them interested in using the system (Froehlich et al., 2012). The system should be made available to members of the household at all times, including allowing them to get some indication of energy 
consumption by simply just passing by the application. Furthermore, the display should be somewhere central in the home, where most members will spend some time each day (Erickson et al., 2013; Fitzpatrick \& Smith, 2009; Froehlich et al., 2012). In terms of aesthetics, it is important that the graphical elements of the system, as well as the physical display, are appealing to the users and fit well with the general décor of the home (Fitzpatrick \& Smith, 2009; Riche et al., 2010).

The user interface for PowerViz consists of four individual screens: current usage (screen saver), usage history, appliance usage, and appliance history.

The current usage screen saver is the only one designed based on artistic visualization, to create ongoing engagement with the system while giving a playful overview of total household energy consumption. When users touch the display, this gives access to the detailed information screens. These screens are designed using a pragmatic visualization approach to foster immediate understanding of information. Whenever historical data is used we showed intervals of 1 hour, 24 hours and 3 days. We intentionally limited the amount of historical data to 3 days to reduce the amount of data that needed to be kept by the system at any given time. Users of the system can move between these three different screens using the standard swipe gesture for tablet computers.

\section{Current Usage}

Current usage, represented on the screen saver, is an abstract visualisation of the current power consumption (figure 1). The screen saver becomes active when the system has not been interacted with for a set amount of time. This was set to 2 minutes during the field trial. When consumption of energy in the whole house rises, the number of lit light globes on the screen increases. When the consumption drops the globes become unlit and fade out, one by one, over time so that people know that something was on, but has since been turned off.

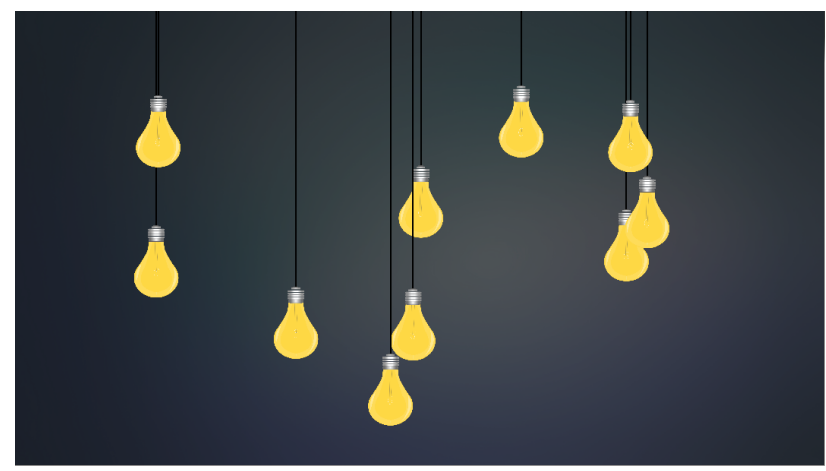

Figure 1. Current Usage (screen saver) indicating total amount of energy used in house.

The main idea behind this screen is to engage users and give the system an ongoing appeal. It should make the members of the household curious about the current energy consumption in the house. They might notice changes in the number of lights and then wonder what that change means. Being an ambient display they can ignore it or notice it as they pass by, registering a number of lit globes, but without any need for further action. Alternatively, they might be motivated to touch the screen and explore the usage data more deeply. The design of the screen, with a black background, and fewer lights as fewer appliances are on, means that it will be less visually dominant at night, when a bright display might disturb.

\section{Usage History}

The householders can view their consumption over time using historical data (figure 2). The time against usage area charts show the total consumption and a comparison of usage patterns for each room in the house.

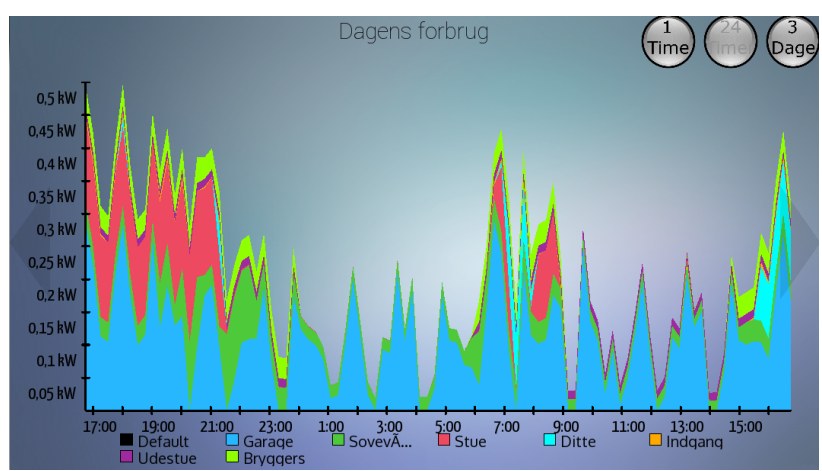

Figure 2. Usage History -

showing consumption over time in each room of the house.

On the area chart, different colours represent the amount of power used in each room in kilowatt-hours. For example, on figure 2 blue is the power used in the garage, green is the power used in bedroom A, and red is the power used in the living room. Users can choose three different graphs, the one-hour history, the last 24 hours of use, and the last three days of use. Different colours are used for the different rooms so that users can easily compare consumption between rooms or get an overview of which rooms have used the most power.

\section{Appliance Usage}

To give the users a better understanding of which appliances consume the most energy, we provide a visualization at appliance level (figure 3 ). We also wanted to study how appliance level information contributes to raising peoples' awareness of their energy consumption behaviours. This is a novel way to represent household consumption, giving users access to much more detailed, fine-grained information than existing home energy management systems.

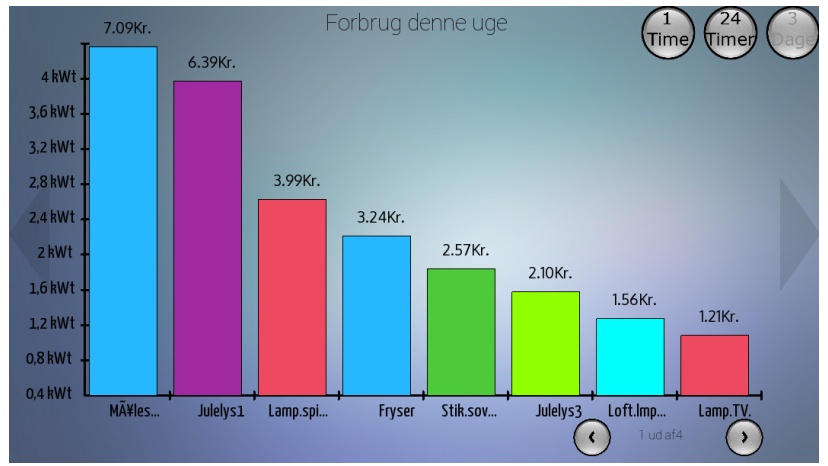

Figure 3. Appliance Usage showing individual appliance energy consumption. 
This screen shows two types of information in a bar chart. The information includes the kilowatts per hour consumed by each device in the house that is plugged in to a power socket, within a specified time period. At the top of each bar, the current cost (in this case in Danish Kroner) of this consumption is calculated and displayed. In the situation where there are too many current devices plugged in to be legibly displayed on one screen, left and right arrows can be used to browse between different devices. The devices are ordered on the chart so that the device currently using the most power will be shown first (in the left most position). The rest of the devices are then shown in decreasing order of electricity consumption. We have assumed that the most power consuming devices will be of most interest to consumers and therefore appear on the chart when this screen opens.

\section{Appliance History}

The appliance history screen is quite different from the others (figure 4). Instead of visualizing consumption measured in kilowatts or price, we show the history of use of an appliance, that is, when it has been turned on or off (and therefore consumed energy) over time. Appliances are shown grouped by rooms of the house, represented in the same colour used for that room in the usage history graph. In this screen, green represents bedroom $\mathrm{A}$, and red represents the living room. This is shown for the last 24-hour period of time, and can be scrolled left or right to see different times of the day.

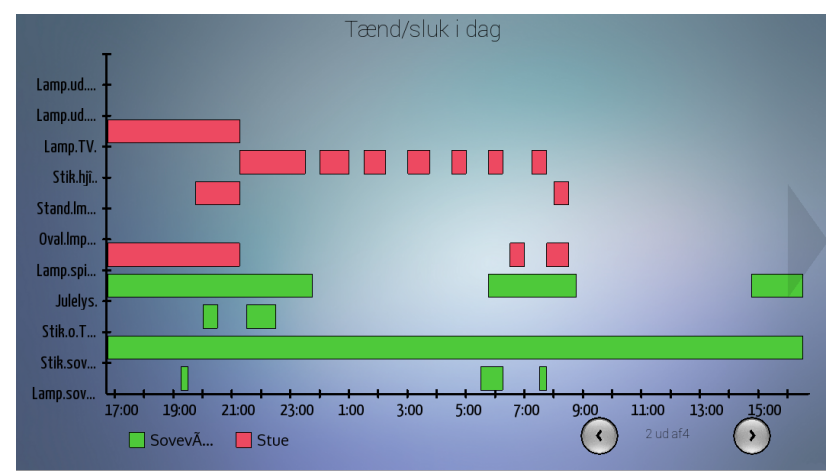

Figure 4. Appliance History showing when appliances are on and off.

This visualization gives consumers an overview of how they use different appliances during the day and over an extended period of time. It also shows them when specific appliances have been turned on and off, making it possible for them to reflect on their behaviours, such as leaving a light on in a room, for example this might happen in a bathroom with no external windows, when it is not even in use.

\section{Implementation}

The PowerViz application was developed in the multi platform programming language $\mathrm{HAXE}^{1}$. This choice allowed us to distribute the application to several platforms at once.

The implementation was split into two major components. It consists of a display application that the

\footnotetext{
${ }^{1}$ The HAXE programming language: http://haxe.org/
}

user interacts with and a backbone server that manages data from different sources (Figure 5). It is the responsibility of the display application to present information to the user and report information about the users interaction with the system. It is the responsibility of the backbone server to retrieve participants' electricity consumption data.

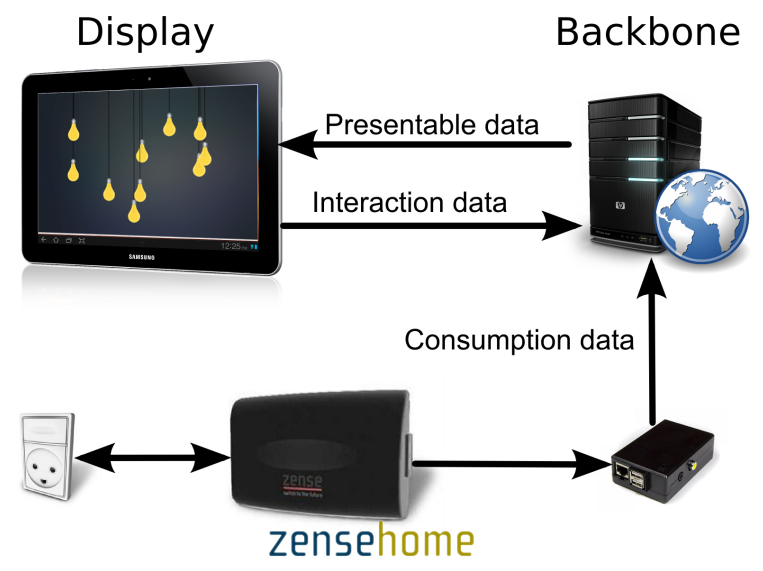

Figure 5. PowerViz system layout.

\section{The Display Application}

The display application consists of two parts: A web application and an Android application. The web application has the responsibility of presenting data to the users, while the Android application encapsulates the web application and shows it on the display tablet. The PowerViz display application also supports user interaction logging. All user interactions are logged and sent to the backbone server. The web application was developed using traditional web technologies such as HTML and CSS, but for structuring the application code TypeScript was chosen instead of regular JavaScript. PowerViz was developed as a web application due to the iterative nature of the system design, making it possible to roll out revisions to the users without manually updating the application on the actual devices located in the households. The application installed on the Android tablet includes special functionality for ensuring alwayson behaviour, making the application turn on the screen and launch itself if the user turns off the screen.

\section{The Backbone Server}

To supply the display application with information, a backbone for handling information was implemented. The main component of the backbone is a web server that retrieves and handles data from the consumption data component. The server also prepares data for the display application, as well as storing logged interaction data.

To obtain electricity consumption data the backbone relies on ZenseHome ${ }^{2}$ system, which is capable of collecting detailed consumption data in near real-time. To retrieve that data a Raspberry $\mathrm{Pi}$ is directly connected to the ZenseHome control box. The ZenseHome control box does not offer the retrieval of consumption data other than the current load on each connected smart power outlet. The connected Raspberry Pi therefore needs to retrieve

\footnotetext{
${ }^{2}$ ZenseHome: http://www.zensehome.dk
} 
data from outlets every two seconds, using the control box as an interface. Since the ZenseHome system is not originally designed for retrieving electricity consumption data so rapidly, techniques for avoiding data collisions were a major focus during the implementation of the system. After data is acquired it is sent to the backbone web server, which then accumulates values and stores historical consumption data in 15-minute intervals.

\section{FIELD STUDY}

There are several well-tested techniques in HCI for studying how users interact with a system design and how this system then affects their behaviours and attitudes. Within qualitative methods, which allows for a smaller cohort of participants and focuses on the quality of the data collected rather than statistical analysis of it, we chose to use the technology probe approach (Hutchinson et al., 2003). We combined this with interviews both during and on conclusion of the field deployment of our prototype system. In this way we were able to gather both the behaviours (logged data) and the reflections of the participants in the study. Given that we focussed on the raise awareness phase of Riche et al.'s (2010) three-stage model, we needed to understand how different aspects of our design, in particular the appliance level representations, achieved this aim in respect to their energy consumption behaviours.

Technology probes present a unique opportunity for gathering data about peoples' everyday life without interfering with their daily routines. The use of probes to explore the domain of domestic sustainable practices has already been used in similar research. Froehlich et al. (2012) used a probe approach for testing alternative designs for their fixture-level water usage display. We designed our technology probe so that data collection would take place when users interacted with a working system. In this way, the probe gathered activity data, which we analysed and used to design the semi-structured interview questions for individual households. We used these interviews to gain insight into people's experiences generally with the system. We also wanted to explore whether people's energy conservation behaviours were affected by knowledge of how individual appliances contributed to the overall electricity consumption.

\section{Participants and Procedure}

Our study included three households from Northern Jutland, Denmark where PowerViz was deployed in each household for a total period of seven weeks. The number of households that could participate was limited, because PowerViz requires that the household already have the ZenseHome system installed, including smart power outlets throughout the house. The participants also had to be willing to have the additional equipment required by the PowerVis system installed, including a display located in a highly visible, high traffic location within their home (figure 6).

All participants had standard household appliances, like fridges, freezers, washing machines, ovens, microwave ovens etc., and also a wide collection of other powerconsuming devices, e.g., laptops, TVs, stereos, gaming consoles. All households generally indicated quite a high awareness of their own power consumption and were generally interested in how and where power was consumed in their households. They originally acquired the ZenseHome technology because it provided flexibility and opportunities for controlling their household appliances.

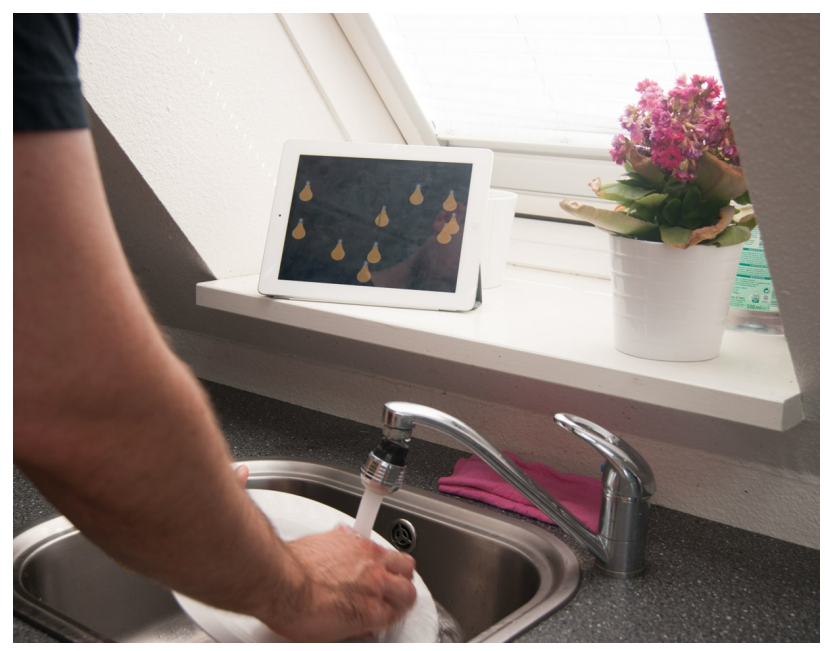

Figure 6. PowerViz display, located in a highly visible, high traffic location in the home.

The three households consisted of the following families:

- Household A: 2 parents (aged between 35-40 years) and 3 young children. They expressed a high awareness of consumption and knew where they used electricity.

- Household B: 2 adults (aged between 35-40 years). They expressed medium awareness of consumption.

- Household C: 2 parents (aged between 45-50 years) and 2 teenaged children. They expressed a high level of awareness of consumption but had to rely on the electricity bill to isolate excessive use.

We implemented certain logging features to access data on people's interactions with the system. This data is stored in a database on a server. Four types of information were logged: consumption data, navigation data, interactions, and maximum watt value.

Consumption data was collected from all devices in the house that were connected to the system via the smart power outlets and through the ZenseHome control box. This data was then used to produce all the power use data presented on the various screens of PowerViz. While the consumption data gives us insight as to how much electricity is being used, this does not tell us about the usage of the system. For research purposes, we also provided a facility to log navigation between the different screens in the application. This tells us which views are being used, the order they are used in, and how often they are being used and for how long. In addition to pure navigation logging we also implemented a feature to tell us when the system goes from an active state to an inactive state (the screen saver). The reason for this is that we want to know when the system is being used, and how much of the time it is displaying the ambient information provided by the light globes interface. In our application householders have the option to set goals about maximum 
energy amounts (in kilo-watts) that they would like to use at different times of the day. They can change this value if they feel that the level has been set too low. We also logged this action to see how their behaviours changed and adapted to their own goals.

In addition to collecting data we implemented functionality in our prototype to provoke users to respond to the system. We did this by changing the number of light globes on the screen saver (acting as an ambient display) to reflect increases and decreases in the overall power consumption of the household. We were then able to see if people were enticed by the growing number of light globes on the screen to check the actual figures on how the power was being used at appliance level. We used this information to ask individual households later in the interviews how they responded to that information, and whether it encouraged them to go and turn off unnecessary appliances.

\section{Qualitative Data Collection and Analysis}

We conducted semi-structured interviews with the three households both in the middle of the field study and at the end of the seven weeks. All interviews took place in participant homes. The sessions were audio recorded. Interviews began with an introduction explaining our interest in the use of PowerViz. We discussed their awareness of their electricity consumption before and after the system was deployed. This included questions about what information was available in the system, how readily available they found it and how they used information about their consumption to make changes in their consumption. We also asked for their general impressions of the system, for example, which screens they used the most and why.

We analysed all interview and data logs using qualitative content analysis with partial transcription to understand in what ways the system had moved the users towards being more aware of their power consumption.

\section{FINDINGS}

We have categorised our findings into four different aspects namely 1) consumption understanding, e.g. how aware they are of their consumption; 2) motivation for energy conservation, e.g. what motivates them to conserve energy; 3) motivation for system use, e.g. what motivates them to use such a system; and 4) system improvements, e.g. what would they want in a system to make it more useful or interesting. We illustrate and explain these in the following sub-sections.

\section{Consumption Understanding}

When asked about how they used their power bill the participants all answered that they used it very little or not at all. The power bill only served as a reminder about how much power they had used over a certain period, but once it is received the power had already been used so they felt unable to do anything about that. As one household said, "the power bill motivates and reminds you of how much you use on power and/or energy in general. If you use too much it is something that will annoy you.",
While they did not use their power bill, they all to some extent used their existing smart outlets. A limitation with these outlets however was that they cannot show the current consumption of the whole household, or grouped as rooms of the house. What they did deliver was current total consumption in kilowatt-hours. Participants said that the PowerViz representations were much more accessible and understandable. They also appreciated the breakdown into appliance level information giving them a better understanding of the contributions of individual appliances to the total amount of household energy consumption. A system that was easier to use and provided more detailed breakdown of information, PowerViz, made them more aware of their consumption.

Most households started with a basic understanding of their consumption. All were engaged in actively conserving energy and this had prompted installing the ZenseHome system and participating in our study. Before our study, they felt they had a good understanding of their household energy consumption but when asked about their consumption habits it became evident that our system had exposed areas of consumption that they were not previously aware of. One participant said that, "sometimes you can get a bit surprised when you do not think that anything is on, but a lot of globes are on, then you look around the house."

Our system assisted them in identifying individual light globes, or other appliances, that they were unaware had been left on. So even though they know their general consumption behaviours, there were still appliances on in the house that surprised them. PowerViz gave them the visualizations to facilitate action to switch off unnecessary appliances. One participant said that he learned how many globes should be on the screen saver when all necessary equipment was on in the house. When more globes appeared than the expected number, it encouraged him to use the system to find out why.

When asked if some individuals in the house consumed more energy than others, and why this might be the case, two reasons were given. Firstly, that the members of the household who use the most simply do not care about consumption, they have other priorities. Secondly, they cannot relate their consumption (in kilowatt hours) to something more material, e.g. money. In all households, the issue of who used the most power was a difficult one because it is difficult to make individuals accountable for the shared consumption of the household.

Participants had different opinions about which types of devices consume the most power. One participant stated that he thought that most power was used to cook and to wash. This, he stated, was because "they are heavy consumers." Another participant said that after she had seen globes on the screen saver turn on when she used the kettle, she looked at the detailed appliance level information and was surprised how much energy the kettle consumed. Some rationalised the use of these "heavy consumers" in terms of the duration of their user, "Well, if it is a relatively short time, you could say, that that would not be the worst consumer." This indicates that, for example, having lights turned on for a long 
period of time might be seen as more of an issue than high consumption spikes by individual appliances. For this kind of information they would check the appliance history (figure 4) to see which devices were on for a long time. Although our appliance history display shows times an appliance is on and off, it does not show this against the accumulated consumption of that device. This could be useful in assisting conservation behaviours when trading off the use of competing appliances.

\section{Motivation for Energy Conservation}

What motivates users into taking action in conserving energy was one of the important parts of this study. In our study households, the main motivation behind conserving energy was, to some extent, to save money. When asked directly, two participants said that although environmental factors were important to them, financial savings was the biggest motivator. Environmental concerns were still very relevant to their usage of the system and they stated that they would take the energy source into consideration, e.g. coal or wind energy, if that information was provided. As one participant said, "Sadly I don't think that far. That's not where my thoughts are at. They are in my right back pocket".

Even though the main motivating factors were economical and environmental concerns, there was a general interest in just saving energy. Interestingly, one participant said his main focus was on limiting consumption and not necessarily saving money, "It's the sport of it, yes. It's nerdy, that's probably what you would call it.". Another participant who claimed no interest in conserving energy at all said she might be motivated to take action, if the environmental consequences of her actions was easily accessible, "It would make a big difference to how much I would think about it, when putting it in perspective to the environment."

Participants from two of the households mentioned more information as a motivating factor for altering consumption behaviour. This would facilitate household members to act according to good environmental behaviour and even exercise some level of flexibility in energy consumption. One participant said, "For example if the globes [on the screen saver] were green, then I would know that now the energy is "green", then I might start the washing machine." Receiving information about the environmental consequences of the current energy supply would make a difference, even to the participant not generally motivated by environmental factors, "If you had an indication of it, then you might say 'I'll allow myself to use the dryer today, then'. There are things that can wait".

\section{Motivation for System Usage}

If the intention of using PowerViz is to make people more aware of their consumption behaviours, then it is also important that we identify what aspects of the design motivated people to use it, and what engaged them into using it for an extended period of time.

The current usage screen (screen saver) was designed in a playful way to specifically lure users into using the system and further exploring the information available.
One of the main problems with the ZenseHome system was that it was too difficult to access the information. PowerViz, in contrast, succeeded in getting people to use the system, even if it was just interpreting what the current screen saver display might mean about their overall consumption. However, this did not always encourage them to take the time to access the more detailed information within the system. Participants stated that they only used other parts of the system a couple of times to identifying problems or explore consumption. One participant mentioned that the main reason for exploring was that they discovered an unexpected number of globes lit on the screen saver, "there was a lot of globes that were on for a longer period of time. This triggered me to go out and look at things." However, participants reported that their interest in the system dropped over time, "It is not as new any more so maybe the interest for it has declined a bit". While the system initially triggered them to explore their consumption, it seems that over time their usage declined. One participant said that this was because they had already learned about their consumption patterns from the system, had adjusted their behaviours to a level they were happy with, and so in the end they more or less used the screen saver as a an always visible confirmation of this level. They would use the system only to check on the details about appliances that created unusual peaks in their overall consumption.

\section{System Improvements}

Participants offered suggestions about how they wanted the system to inform them and how they imagined such information could be presented. A common desire was the possibility to get usage statistics for more than just three days. Although the three day usage overview was useful, being able to get statistics for an entire week seemed more naturally in tune with household living patterns. Being able to view consumption data for an entire month would also make it easier to compare detailed consumption information against the power bill. Comparing consumption in different months would make it easier to identify annual consumption patterns.

Participants also thought that a more active system which issues notifications about abnormalities in consumption would be useful, so that when consumption occurs that does not match normal behaviour, they would know about it immediately. In respect to declining use of the system over time, participants said that the system should do something else in addition to just showing consumption data. One said that it might be an idea to shuffle the screen saver and the other screens and show them randomly for a period of time. Another said that he wanted completely different information such as news or weather information appearing on the screen saver. These types of improvements would make users pay more attention to the system as they go about their daily routines. One user put forward the idea of viewing his consumption based on activities instead of rooms. Offering consumption information based on activities would make it easier to make a connection between actions taken and energy consumed, "If you organized it as categories; lights, freezer and so on - then you would be able to see if something went out of control." 


\section{DISCUSSION}

Through our interviews we found that participants generally had a good understanding of power consumed in their home. We think that already having a smart home solution supplied by a Danish manufacturer influenced this and gave these households much better access to information about their consumption than the monthly power bill or meters in a cupboard that other households rely on.

Interestingly, householder's understanding of their consumption was that they had a base level that was unavoidable and a consumption beyond that which could be changed. Most of the base level consumption was seen as shared among all members of the household, and even parts of the non-base level consumption were considered shared. This non-base level consumption is the part that is open for negotiation and reduction as a group. We believe that a system with information that could support this process would be very useful to householders, and to some extent our appliance level information does help here when identifying non-essential appliances that are high consumers. It seems that from their responses to changes in the screen saver, householders do not take long to decide what is a "normal" operating level of energy consumption in their home. Their attention can then be drawn to active appliances outside of this norm.

\section{Motivation for Electricity Saving}

Motivation to use the PowerViz system was initially the novelty of the system. Not surprisingly, this declined over time. In the end, the screen saver with the light globes became the main point of reference. When an unexpected number of light globes were displayed on the screen saver participants would become curious and explore the system further. They did this to find explanations for the change, most often for an increase in the number of light globes. This success of the ambient display (figure 1) was very encouraging. This is both in terms of people using it to keep an eye on things, and from our point of view, as an encouragement for users to dig deeper into the details of the system. All participants preferred our system for viewing detailed consumption information compared to their existing complex smart home energy management software. The PowerViz eco-feedback system raised awareness about their energy consumption. This was both in terms of overall consumption, e.g. being surprised when an unusual number of lit globes were displayed, and in terms of the contribution of specific appliances to the overall consumption.

The motivating factors for energy conservation were mainly financial, but environmental concerns also played a role. Users expressed that they would respond positively and adjust their behaviour if they had easy access to information about the wider environmental consequences of their energy consumption. We believe that finding new, poignant and creative ways to represent financial gains or comparisons of energy sources in the system would further motivate energy saving behaviour. Designs should go beyond the simple numeric listing of costs against usage data that we used in the appliance usage bar chart (figure 3), which did not actively assist people in making decisions about how to save money. It would also be interesting to have a system that makes it possible to target the non-base level consumption of a household. Our appliance level representation (figure 3) goes part way towards providing information to support the kinds of negotiations needed to target non-base level devices, but relies on human agency to make comparisons across all devices. It would be good to provide a facility for user-defined groupings of devices, so that they could match the household's view of them. Participants had already shared that they would like the system to present information in ways beyond the current screens. Although other or additional information presented on the screen saver was the most popular recommendation from participants, we believe that there is still plenty of opportunity for exploring alternative designs for the different detailed information screens.

\section{Limitations}

Our study is characterized by a number of limitations that affect the generalizability of our findings. As already noted, we could only include participants and households with ZenseHome technology already installed as we were interested in the appliance level consumption. As a direct consequence, our households (in particular the adult members) were already highly aware of their own power consumption and probably more aware than the average electricity consumer in Denmark, and they were also quite concerned with respect to how they could reduce or save electricity. Also, number of participating households $(\mathrm{N}=3)$ is quite low for such studies. However, we believe that some of our qualitative findings could be useful for further studies and research for appliance level electricity consumption.

Our study was conducted in Denmark and therefore some of the findings may be challenging to generalize beyond consumption awareness in Denmark. Different countries have different opportunities and obstacles for introducing power saving mechanisms, e.g. few Danish households have air-conditioning due to weather conditions, but some Danish households have electricity powered heating.

\section{CONCLUSIONS}

In this paper, we presented the design and field study of an appliance level eco-feedback system, PowerViz. We created the design based on principles from research in this area. The system draws its data from a smart home energy metering system, obtaining usage data from each outlet in the household. We used the technology probe approach with follow-up semi-structured interviews to identify the impact of our system on consumer awareness towards energy consumption.

We presented a set of findings from the field study that we classified as either relating to awareness of consumption, motivation for using the system, motivation for conserving energy or system specific improvement proposals.

We found that having PowerViz in their home did increase the participants' awareness of their own energy consumption. The system provided a set of interfaces through which they could identify specific devices that 
used more energy than expected. We also found that even though we designed a set of information visualizations for exploring detailed consumption information, participants most often used the information displayed on the screen saver as an ambient information source. We confirmed that people's main motivation for conserving energy is financial savings, although information about the environmental consequences of consumption seems to have a positive effect on people's conservation of energy. Participants were generally open towards being more flexible in their consumption behaviour, if the system could help them to do so.

Our own future work includes further development of our appliance level eco-feedback system and further longitudinal studies. The aim of this research is to study not only consumption awareness, but also flexibility in electricity usage towards shifting parts of one's electricity usage to times when it is either produced from green power sources (e.g. wind or solar power), or when there is an abundance of capacity in the electricity network.

\section{ACKNOWLEDGMENTS}

This work was partially funded by the TotalFlex project. www.totalflex.dk. We thank all participating families.

\section{REFERENCES}

Cowan, B. R., Bowers, C. P., Beale, R. and Pinder, C. The stroppy kettle: An intervention to break energy consumption habits. In Proc. Ext. Abstracts CHI 2013, ACM (2013), 1485-1490.

Darby, S. The effectiveness of feedback on energy consumption. Tech. rep. DEFRA, 2006.

DiSalvo, C., Sengers, P. and Brynjarsdottir, H. Mapping the landscape of sustainable hci. In Proc. CHI 2010, ACM (2010), 1975-1984.

EIA (U.S. Energy Information Administration). Residential Energy Consumption Survey (2013). http://www.eia.gov/tools/faqs/faq.cfm?id=86\&t $=1$

Erickson, T., Li, M., Kim, Y., Deshpande, A., Sahu, S., Chao, T., Sukaviriya, P. and Naphade, M. The Dubuque Electricity Portal: Evaluation of a city-scale residential electricity consumption feedback system. In Proc. CHI 2013, ACM (2013), 1203-1212.

Fitzpatrick, G. and Smith, G. Technology-enabled feedback on domestic energy consumption: Articulating a set of design concerns. Pervasive Computing 8, 1 (2009), 37-44.

Foster, D., Linehan, C., Lawson, S., and Kirman, B. Power ballads: Deploying aversive energy feedback in social media. In Proc. Ext. Abstracts CHI 2011, ACM Press (2011), 2221-2226.

Froehlich, J. Promoting energy efficient behaviors in the home through feedback: The role of human computer interaction. In Proc. HCIC 2009 Winter Workshop (2009).

Froehlich, J., Findlater, L., and Landay, J. The design of eco-feedback technology. In Proc. CHI 2010, ACM Press (2010), 1999-2008.
Froehlich, J., Findlater, L., Ostergren, M., Ramanathan, S., Peterson, J., Wragg, I., Larson, E., Fu, F., Bai, M., Patel, S. and Landay, J. A. The design and evaluation of prototype eco-feedback displays for fixture-level water usage data. In Proc. CHI 2012, ACM Press (2012), 2367-2376.

Heller, F. and Borchers, J. PowerSocket: Towards onoutlet power consumption visualization. In Proc. Ext. Abstracts CHI 2011, ACM Press (2011), 1981-1986.

Hutchinson, H., Mackay, W., Westerlund, B., Bederson, B. B., Druin, A., Plaisant, C., Beaudouin-Lafon, M., Conversy, S., Evans, H., Hansen, H., Roussel, N. and Eiderback, B. Technology probes: Inspiring design for and with families. In Proc. CHI 2003, ACM Press (2003), 17-24.

Kim, T., Hong, H. and Magerko, B. Coralog: Use-aware visualization connecting human micro-activities to environmental change. In Proc. Ext. Abstracts CHI 2009, ACM Press (2009), 4303-4308.

Kjeldskov, J., Skov, M. B., Paay, J. and Pathmanathan, R. Using mobile phones to support sustainability: A field study of residential electricity consumption. In Proc. CHI 2012, ACM Press (2012), 2347-2356.

Mankoff, J. C., Blevis, E., Borning, A., Friedman, B., Fussell, S. R., Hasbrouck, J., Woodruff, A., and Sengers, P. Environmental sustainability and interaction. In Proc. Ext. Abstracts CHI 2007, ACM Press (2007), 2121-2124.

Milenkovic, M., Hanebutte, U., Huang, Y., Prendergast, D. and Pham, H. Improving user comfort and office energy efficiency with poem (personal office energy monitor). In Proc. Ext. Abstracts CHI 2013, ACM Press (2013), 1455-1460.

Petkov, P., Kobler, F., Foth, M., Medland, R. and Krcmar, H. Engaging energy saving through motivation-specific social comparison. In Proc. Ext. Abstracts CHI 2011, ACM Press (2011), 1945-1950.

Pierce, J., Odom, W. and Blevis, E. Energy aware dwelling: A critical survey of interaction design for eco-visualizations. In Proc. $\mathrm{OzCHI}$ 2008, ACM Press (2008), 1-8.

Riche, Y., Dodge, J. and Metoyer, R. A. Studying always-on electricity feedback in the home. In Proc. CHI 2010, ACM Press (2010), 1995-1998.

Schwartz, T., Denef, S., Stevens, G., Ramirez, L. and Wulf, V. Cultivating energy literacy: Results from a longitudinal living lab study of a home energy management system. In Proc. CHI 2013, ACM Press (2013), 1193-1202.

Snow, S., Buys, L., Roe, P. and Brereton, M. Curiosity to cupboard- self reported disengagement with energy use feedback over time. In Proc. OzCHI 2013, ACM Press (2013), 245-254.

World Energy Council. Energy Efficiency Indicators by Country/Region. Energidata (2014) http://www.worldenergy.org/data/efficiency-indicators/ 